\title{
Blood Pressure Variability in Acute Ischemic Stroke: The Role of Early Recanalization
}

\author{
Ana Inês Martins ${ }^{a}$ João Sargento-Freitas ${ }^{\text {a, e } \quad J o a n a ~ J e s u s-R i b e i r o ~}{ }^{a}$ \\ Inês Correia $^{a}$ Leila Cardoso $^{b}$ João Pedro Gomes ${ }^{b}$ Mariana Gonçalves ${ }^{b}$ \\ Rui Costa ${ }^{c}$ Fernando Silva $^{a}$ Orlando Galego $^{d}$ César Nunes $^{d}$ \\ José Beato-Coelho ${ }^{a}$ Ricardo Varela ${ }^{a}$ Cristina Machado ${ }^{a}$ Bruno Rodrigues $^{a}$ \\ Gustavo C. Santo ${ }^{a}$ Luís Cunhaa,e \\ ${ }^{a}$ Department of Neurology, Centro Hospitalar e Universitário de Coimbra, Coimbra, Portugal; ${ }^{\mathrm{b}}$ Department of \\ Internal Medicine, Centro Hospitalar e Universitário de Coimbra, Coimbra, Portugal; ' Department of Internal \\ Medicine, Tondela-Viseu Hospital Center, Coimbra, Portugal; ${ }^{d}$ Department of Neuroradiology, Centro Hospitalar e \\ Universitário de Coimbra, Coimbra, Portugal; ${ }^{\text {F }}$ aculty of Medicine, Coimbra University, Coimbra, Portugal
}

\section{Keywords}

Blood pressure fluctuations · Recanalization · Acute stroke

\begin{abstract}
We performed a retrospective study with the aim of investigating the association between blood pressure (BP) variability in the first $24 \mathrm{~h}$ after ischemic stroke and functional outcome, regarding arterial recanalization status. A total of 674 patients diagnosed with acute stroke and treated with revascularization therapies were enrolled. Systolic and diastolic $\mathrm{BP}$ values of the first $24 \mathrm{~h}$ after stroke were collected and their variation quantified through standard deviation. Recanalization state was evaluated at $6 \mathrm{~h}$ and clinical outcome at 3 months was assessed by modified Rankin Scale. In multivariate analyses systolic BP variability in the first $24 \mathrm{~h}$ poststroke showed an association with 3 months clinical outcome in the whole population and non-recanalyzed patients. In recanalyzed patients, BP variability did not show a significant association with functional outcome.
\end{abstract}

(c) 2018 S. Karger AG, Basel

\section{KARGER}

(c) 2018 S. Karger AG, Basel

E-Mail karger@karger.com

www.karger.com/ene

\section{Introduction}

Blood pressure (BP) is an independent predictor of functional outcome in acute ischemic stroke setting [1]. It has been consistently reported that higher systolic and diastolic BP values (SBP and DBP) are related to worst outcomes. Spontaneous variations in SBP and DBP in the acute phase after ischemic stroke are common, however their pathological background and clinical impact are still largely undetermined. Previous work suggested that BP variability in the acute phase might be linked to a worst prognosis, by enhancing symptomatic intracerebral hemorrhage, early neurological deterioration, and death rates after treatment with intravenous recombinant tissue plasminogen activator (IVrtPA) [2]. Recent work from our group demonstrated that early recanalization status influences directly the association of $24 \mathrm{~h} \mathrm{BP}$ and clinical outcome [3]. Nevertheless, studies assessing the effect of acute cerebrovascular hemodynamic status on the clinical impact of spontaneous BP fluctuations are lacking.

João Sargento-Freitas

Department of Neurology

Centro Hospitalar e Universitário de Coimbra

PT-3000-075 Coimbra (Portugal)

E-Mail jsargentof@hotmail.com 
Table 1. Baseline characteristics of the study, univariate linear regression for predictors of SBP and DBP fluctuations at $24 \mathrm{~h}$

\begin{tabular}{|c|c|c|c|c|c|}
\hline Variable & $\begin{array}{l}\text { Total population } \\
n=674 \text { patients }\end{array}$ & $\begin{array}{l}\text { Univariate association } \\
\text { with SBP SD, B (95\% CI) }\end{array}$ & $p$ value & $\begin{array}{l}\text { Univariate association } \\
\text { DBP SD, B }(95 \% \text { CI })\end{array}$ & $p$ value \\
\hline Age, years, mean $\pm S D$ & $73.28 \pm 11.50$ & $0.083(0.030$ to 0.136$)$ & 0.002 & $0.040(0.004$ to 0.076$)$ & 0.029 \\
\hline Gender, male, $n(\%)$ & $363(53.90)$ & $-0.517(-1,738$ to 0.704$)$ & 0.406 & $-0.286(-1.120$ to 0.548$)$ & 0.501 \\
\hline Smoking, $n(\%)$ & $67(9.90)$ & $0.890(-1.142$ to 2.922$)$ & 0.390 & $0.718(-0.675$ to 2.110$)$ & 0.312 \\
\hline Alcoholism, $n(\%)$ & $45(6.70)$ & $0.115(-1.059$ to 1.289$)$ & 0.975 & $-0.051(-0.806$ to 0.724$)$ & 0.876 \\
\hline Hypertension, $n(\%)$ & $541(80.40)$ & $1.818(0.290$ to 3.346$)$ & 0.020 & $0.751(-0.296$ to 1.798$)$ & 0.159 \\
\hline Diabetes mellitus, $n(\%)$ & $166(24.60)$ & $1.510(0.099$ to 2.921$)$ & 0.006 & $0.867(-0.113$ to 1.847$)$ & 0.083 \\
\hline Dyslipidemia, $n(\%)$ & $353(52.70)$ & $-0.614(-1.837$ to 0.609$)$ & 0.324 & $-0.465(-1.301$ to 0.370$)$ & 0.275 \\
\hline Atrial fibrillation, $n(\%)$ & $329(48.90)$ & $0.137(-1.081$ to 1.355$)$ & 0.826 & $1.724(0.902$ to 2.546$)$ & $<0.001$ \\
\hline Heart failure, $n(\%)$ & $90(13.00)$ & $0.006(-0.002$ to 0.013$)$ & 0.406 & $0.003(-0.002$ to 0.007$)$ & 0.296 \\
\hline Coronary artery disease, $n(\%)$ & $62(9.40)$ & $1.763(-0.343$ to 3.868$)$ & 0.101 & $0.080(-1.362$ to 1.522$)$ & 0.913 \\
\hline Previous mRS, mean \pm SD & $0.31 \pm 0.60$ & $0.972(-0.036$ to 1.980$)$ & 0.059 & $0.351(-0.339$ to 1.041$)$ & 0.318 \\
\hline NIHSS at admission, mean SD & $15.29 \pm 7.01$ & $-0.006(-0.093$ to 0.081$)$ & 0.893 & $0.011(-0.049$ to 0.070$)$ & 0.723 \\
\hline Fibrinolysis (i.v.), $n(\%)$ & $663(98.40)$ & $4.399(-0.394$ to 9.193$)$ & 0.720 & $0.793(-2.490$ to 4.076$)$ & 0.635 \\
\hline Endovascular treatment, $n(\%)$ & $52(7.70)$ & $-1.957(-4.234$ to 0.320$)$ & 0.092 & $-0.069(-1.628$ to 1.490$)$ & 0.931 \\
\hline Time to treatment, min, mean $\pm \mathrm{SD}$ & $150.25 \pm 55.27$ & $0.006(-0.005$ to 0.017$)$ & 0.287 & $-0.007(-0.031$ to 0.018$)$ & 0.605 \\
\hline
\end{tabular}

mRS, modified Rankin Scale; SBP, systolic blood pressure; DBP, diastolic blood pressure; i.v., intravenous.

The main objective of this study is to evaluate the clinical effect of acute BP variability after ischemic stroke in patients submitted to intravenous and/or intra-arterial recanalization treatments, according to recanalization status.

\section{Material and Methods}

We included consecutive patients with acute ischemic stroke from July 2009 to June 2015, submitted to intravenous thrombolysis (IVrtPA) and/or intra-arterial therapies in our tertiary hospital. Vascular risk factors were collected from a standardized local clinical registry. All patients were admitted to the stroke unit and BP readings were performed by protocol every $2 \mathrm{~h}$ during daytime and every $3 \mathrm{~h}$ throughout nighttime. Baseline measurements were obtained previous to recanalization therapies. All BP measurements were performed by certified and trained nurses, in Phillips Agilent NBP equipment, model M1008B, validated annually (last validation in January 2017). Standard deviation (SD) of the first $24 \mathrm{~h}$ systolic BP (SBP SD) and diastolic BP (DBP SD) values were calculated, in order to assess $\mathrm{BP}$ variation in acute phase.

Neurological status at admission was evaluated by using $\mathrm{Na}$ tional Institute of Health Stroke Scale (NIHSS). Clinical data from follow-up appointment (modified Rankin Scale [mRS]) or information obtained by telephone contact were used to estimate 3-month clinical outcome. Arterial recanalization was assessed by angiographic pattern at the end of intra-arterial treatment, cerebral CT angiography, or by transcranial color-coded Doppler performed at $6 \mathrm{~h}$ after stroke. We considered recanalization as grade $2 \mathrm{~b}$ or 3 from the modified Thrombolysis in Cerebral Infarction score in patients under intra-arterial treatment, grades 4 or 5 from the thrombolysis in brain ischemia [4] classification using TCCD, or visualization of contrast perfusion in all symptomatic vessels by CT angiography.

Our institution's protocol regarding BP values in acute phase post-ischemic stroke follows ASA/AHA guidelines, and BP values are kept $<185 \mathrm{~mm} \mathrm{Hg} \mathrm{SBP}$ and $<110 \mathrm{~mm} \mathrm{Hg} \mathrm{DBP} \mathrm{before} \mathrm{intrave-}$ nous and/or intra-arterial therapies and $<180 / 105 \mathrm{~mm} \mathrm{Hg}$ for the first $24 \mathrm{~h}$ after treatment [5].

Symptomatic intracerebral hemorrhage (sICH) defined according to NINDS trial criteria was any intracerebral hemorrhage with neurological deterioration (increase of $\geq 1$ in the NIHSS score) from baseline or death within $36 \mathrm{~h} \mathrm{[6].}$

Considering the observational and retrospective design of the study, a specific signed informed consent was not obtained. All patients received oral and written information regarding collection of observational data for clinical studies and were free to withdraw consent. The institutional Ethics Committee of our hospital approved the study.

Univariate associations were performed with $24 \mathrm{~h}$ SBP SD, DBP $\mathrm{SD}, 3$ months $\mathrm{mRS}$, and sICH, using a linear regression. Ordinal multivariate regression models identified the relationship between BP SD and functional outcome. The variables included in the multivariate model were SBP and DBP SD, and variables with univariate association with SBP SD and DBP SD (Table 1) and sICH and mRS at 3 months (Table 2).

The relationship between of SBP and DBP fluctuations in the first $24 \mathrm{~h}$ post-ischemic stroke and $\mathrm{sICH}$ was determined using a binary logistic regression adjusting for recanalization status and variables with univariate association with SBP SD and DBP SD of the first $24 \mathrm{~h}$.

Statistical significance was set for $p<0.05$. 

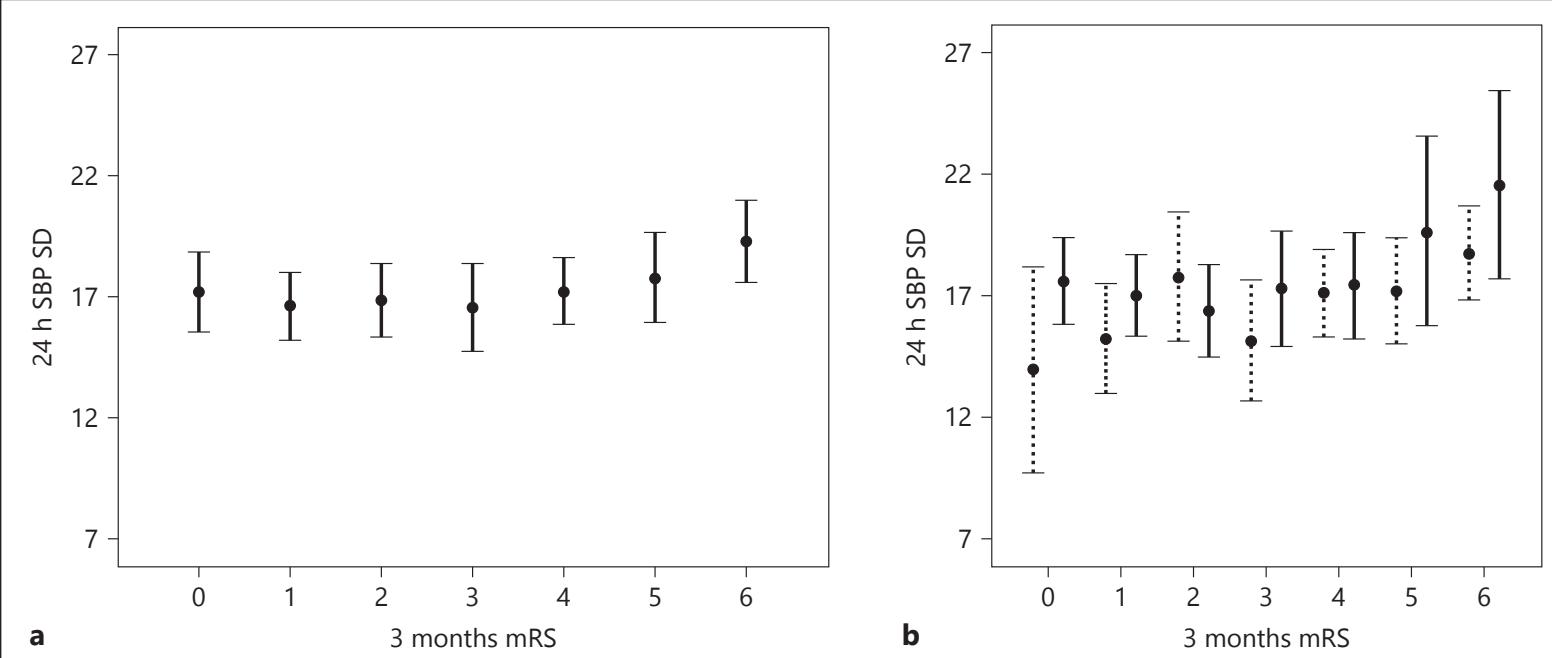

Recanalization at $6 \mathrm{~h}$ post-stroke

i...... No $\longmapsto$ Yes
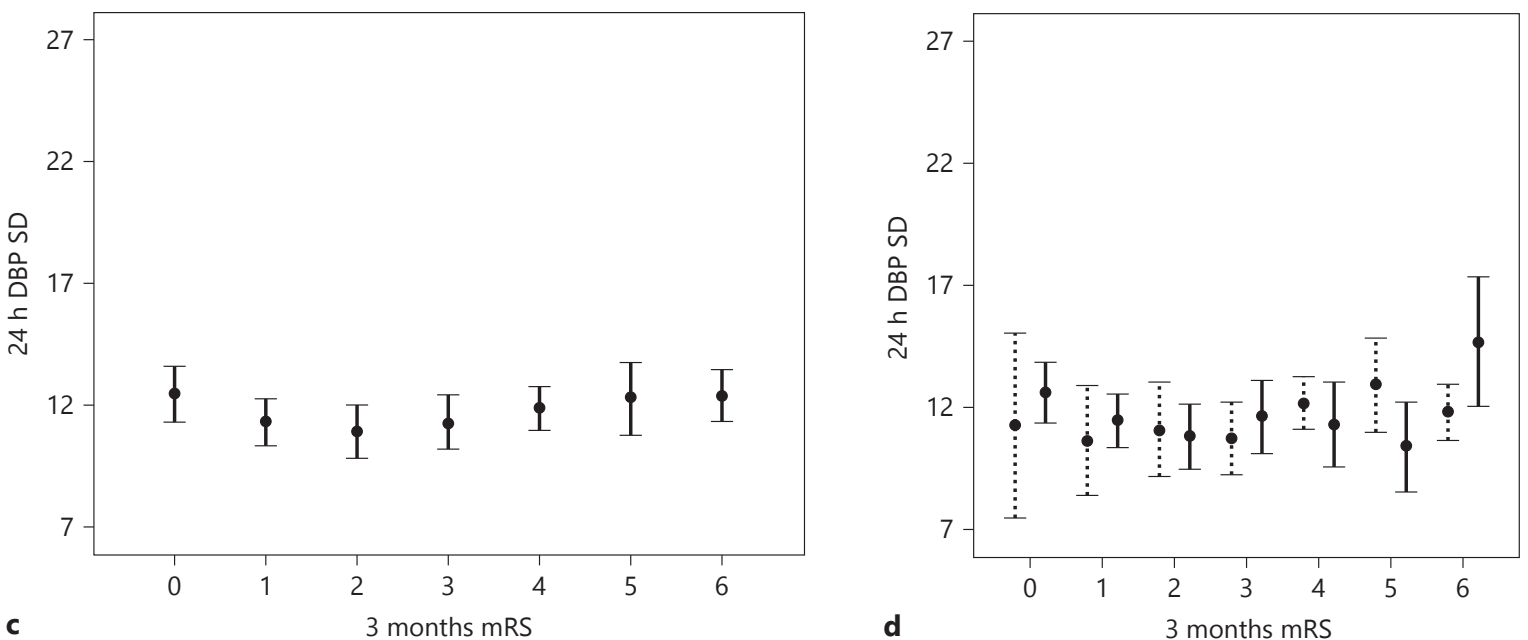

Recanalization at $6 \mathrm{~h}$ post-stroke 1...... No

Fig. 1. Error bar representing the relationship between blood pressure fluctuations in the first $24 \mathrm{~h}$ post-ischemic stroke and functional outcome at 3 months. (a, c) Total population; (b, d) recanalyzed and non-recanalyzed patients. mRS, modified Rankin Scale; BP, Blood pressure; SD, standard deviation; error bars represent the $95 \%$ confidence interval for mean.

\section{Results}

A total of 748 patients were submitted to acute recanalization treatment for ischemic stroke, during the study period, and 674 were enrolled in this study. From the 74 excluded patients, 55 had a previous mRS ranking higher than 2 , and the remaining patients were excluded due to lacking information regarding recanalization status or clinical outcome. Population characteristics are described in Table 1. According to the Oxfordshire Community Stroke Project Classification [7], 442 patients were catalogued as total anterior circulation infarct (TACI), 162 as partial anterior circulation infarct (PACI), 53 as posterior circulation infarct, and 17 as lacunar infarct. Of those, 616 patients had an anterior circulation (carotid) ischemic stroke and 58 were diagnosed with vertebro-basilar circulation ischemic stroke. A total of 663 patients underwent IVrtPA, 52 were submitted to endovascular procedures (11 of which isolated), and recanalization was accomplished in 355 patients (52.70\%). Recanalization was assessed by TCCD in 615 patients (91.20\%), angiography in $52(7.70 \%)$, and CT angiography in $7(1.10 \%)$. A total of 98 patients $(14.50 \%)$ received antihypertensive treatment (labetalol) within the first $24 \mathrm{~h}$.

In our population, sICH was observed in 42 patients (6.20\%). SBP and DBP variation in the first $24 \mathrm{~h}$ was not associated with sICH. (OR 1.023; 95\% CI 0.987-1.061; $p=0.215$ and $\mathrm{OR}, 1.005 ; 95 \%$ CI $0.974-1.037 ; p=0.765)$. 
Table 2. Univariate linear regression for predictors of sICH and 3-month mRS

\begin{tabular}{lcccr}
\hline Variable & $\begin{array}{l}\text { Univariate association with } \\
\text { sICH, B (95\% CI) }\end{array}$ & $p$ value & $\begin{array}{l}\text { Univariate association with } \\
\text { 3 months mRS, B (95\% CI) }\end{array}$ & $p$ value \\
\hline Age, years & $0.001(-0.001$ to 0.002$)$ & 0.311 & $0.050(0.037$ to 0.063$)$ & $<0.001$ \\
Gender, male & $0.026(-0.011$ to 0.063$)$ & 0.162 & $-0.503(-0.811$ to -0.196$)$ & 0.001 \\
Smoking & $-0.036(-0.098$ to 0.025$)$ & 0.244 & $-1.194(-1.703$ to -0.684$)$ & $<0.001$ \\
Alcoholism & $-0.010(-0.045$ to 0.026$)$ & 0.596 & $-0.089(-0.388$ to 0.210$)$ & 0.558 \\
Hypertension & $0.021(-0.025$ to 0.067$)$ & 0.372 & $0.559(0.171$ to 0.946$)$ & 0.005 \\
Diabetes mellitus & $0.010(-0.034$ to 0.053$)$ & 0.665 & $-0.026(-0.391$ to 0.339$)$ & 0.890 \\
Dyslipidemia & $-0.049(-0.085$ to -0.012$)$ & 0.009 & $-0.335(-0.645$ to -0.026$)$ & 0.034 \\
Atrial fibrillation & $0.027(-0.010$ to 0.063$)$ & 0.152 & $0.762(0.458$ to 1.066$)$ & $<0.001$ \\
Heart failure & $0.002(-0.064$ to 0.034$)$ & 0.204 & $0.001(-0.001$ to 0.003$)$ & 0.322 \\
Coronary artery disease & $0.037(-0.027$ to 0.101$)$ & 0.260 & $0.306(-0.228$ to 0.840$)$ & 0.261 \\
Previous mRS & $-0.009(-0.039$ to 0.022$)$ & 0.582 & $0.831(0.582$ to 1.080$)$ & $<0.001$ \\
NIHSS at admission & $0.002(0.000$ to 0.005$)$ & 0.080 & $0.157(0.138$ to 0.175$)$ & $<0.001$ \\
Fibrinolysis (i.v.) & $0.063(-0.081$ to 0.208$)$ & 0.389 & $-0.352(-1.572$ to 0.868$)$ & 0.571 \\
Endovascular treatment & $0.058(-0.011$ to 0.126$)$ & 0.100 & $0.758(0.181$ to 1.334$)$ & 0.098 \\
Time to treatment & $0.001(0.000$ to 0.002$)$ & 0.062 & $0.009(0.001$ to 0.017$)$ & 0.145 \\
\hline
\end{tabular}

mRS, modified Rankin Scale; SBP, systolic blood pressure; DBP, diastolic blood pressure; i.v., intravenous; sICH, symptomatic intracerebral hemorrhage.

Table 3. Multivariate association analyzing the relationship between $24 \mathrm{~h}$ post-ischemic stroke blood pressure fluctuations and 3-month mRS in total population, non-recanalyzed, and recanalyzed patients

\begin{tabular}{lll}
\hline Variable & OR $(95 \% \mathrm{CI})$ & $p$ value \\
\hline SBP SD 24 h total population & $1.023(1.005-1.040)$ & 0.010 \\
DBP SD 24 h total population & $1.008(0.983-1.033)$ & 0.533 \\
SBP SD 24 h non-recanalyzed at 6 h & $1.027(1.001-1.053)$ & 0.041 \\
DBP SD 24 h non-recanalyzed at 6 h & $1.012(0.779-1.049)$ & 0.528 \\
SBP SD 24 h recanalyzed at 6 h & $1.021(0.970-1.046)$ & 0.089 \\
DBP SD 24 h recanalyzed at 6 h & $0.904(0.631-1.027)$ & 0.600 \\
\hline
\end{tabular}

mRS, modified Rankin Scale; SBP, systolic blood pressure; DBP, diastolic blood pressure.

In multivariate analyses, SBP SD in the first $24 \mathrm{~h}$ poststroke was associated with 3 months mRS in total population and in the non-recanalyzed patients. In the recanalyzed group, there was no statistically significant association. DBP SD in the first $24 \mathrm{~h}$ was not associated with 3 months mRS (Fig. 1; Table 3). A sub-analysis including solely TACI and PACI patients revealed similar results.

\section{Discussion}

The main finding of our study is that recanalization status influences the association between SBP and DBP spontaneous fluctuations in the first $24 \mathrm{~h}$ post-ischemic stroke and functional outcome at 3 months. In the non- recanalyzed group, the highest SBP SD values were associated with worst clinical outcomes at 3 months. When early recanalization was accomplished, neither SBP SD nor DBP SD values influenced 3 months functional outcome.

$\mathrm{BP}$ variability has been identified as an independent risk factor for ischemic and hemorrhagic stroke. However, data on the impact of BP fluctuations in acute ischemic stroke are scarce and conflicting $[1,8]$, notwithstanding their high frequency in acute phase. Although the pathophysiologic mechanism is still unsettled, changes in the neuroendocrine system (hypothalamic-pituitary-adrenocortical axis and sympathetic nervous system) and malfunction of cardiac baroreceptor reflex have been proposed to be associated with BP changes in acute ischemic stroke [9]. Moreover, known circadian BP changes could have an impact 
on clinical outcome. However, the profile of nocturnal BP dipping in acute ischemic stroke did not seem to influence functional prognosis. Nonetheless, reverse dipping - a rise in mean nocturnal SBP as compared to mean diurnal SBP - was associated to more frequent hemorrhagic transformations, probably related to reperfusion injury [10].

Early recanalization has been acknowledged as one of the most important predictors of good clinical outcome [11], influencing the association between BP values in the first $24 \mathrm{~h}$ and functional outcome [3]. In our study, SBP fluctuations had a deleterious effect only in the nonrecanalyzed patients, independently from the development of sICH, suggesting an association with the increased hemodynamic stress due to BP variability in patients without early recanalization. Thus, in the nonrecanalyzed patients, penumbra area is rendered with poor autoregulation, remaining directly dependent on collateral circulation. Moreover, when the protective autoregulation mechanisms are malfunctioning, BP changes may result in hyper or hypoperfusion of the ischemic tissue. On the other hand, if early recanalization is accomplished, brain tissue autoregulation is reassured, and BP fluctuations deleterious effects are prevented.

Although spontaneous fluctuations of SBP were associated with functional outcome, DBP SD did not show any association. DBP is related to the relaxing diastolic phase, while SBP is associated to ventricular ejection phase [12], rendering perfusion intensity, which may translate into reperfusion injury. That probably explains why only SBP SD was associated with a poorer prognosis in non-recanalyzed patients, and DBP SD did not have an association with clinical prognosis.
This novel information is of particular importance in current stroke unit care, especially with the widespread use of intravenous and intra-arterial reperfusion therapies and easy access to regular hemodynamic assessments. Furthermore, our findings reinforce that future trials addressing BP management in acute stroke should take recanalization status under consideration.

This study has some limitations. Though it is retrospective, the studied variables are objective and registered prospectively, reducing a possible influence. Moreover, although it is a single-centered study, the demographic and baseline characteristics of the study population were similar to other large stroke cohorts. Additionally, our study design does not albeit identification of the relative contribution of spontaneous vs. therapeutic BP fluctuations, as $14.50 \%$ of the included patients received IV BP lowering treatments. Despite recanalization state being graded by heterogeneous procedures, all methods have been previously validated and performed by experienced vascular neurologists and neuroradiologists.

\section{Acknowledgments}

The authors would like to recognize the collaboration of our Stroke unit's nursing staff for the BP collection.

\section{Disclosure Statement}

J.S.-F. received a speaking fee from Bioportugal in symposium on the subject, "BP in acute stroke: from physiopathology to clinical impact." The remaining authors have nothing to disclose.

\section{References}

1 Willmot M, Leonardi-Bee J, Bath PM: High blood pressure in acute stroke and subsequent outcome a systematic review. Hypertension 2004;43:18-24

2 Endo K, Kario K, Koga M, Nakagawara J, Shiokawa Y, Yamagami $\mathrm{H}$, et al: Impact of early blood pressure variability on stroke outcomes after thrombolysis: The SAMURAI rtPA registry. Stroke 2013;44:816-818.

3 Martins AI, Sargento-Freitas J, Silva F, JesusRibeiro J, Correia I, Gomes JP, et al: Recanalization modulates association between blood pressure and functional outcome in acute ischemic stroke. Stroke 2016;47:15711576.

4 Demchuk AM, Burgin WS, Christou I, Felberg RA, Barber PA, Hill MD, et al: Thrombolysis in brain ischemia (TIBI) transcranial Doppler flow grades predict clinical severity, early recovery, and mortality in patients treat- ed with intravenous tissue plasminogen activator. Stroke 2001;32:89-93.

5 Powers WJ, Rabinstein AA, Ackerson T, Adeoye OM, Bambakidis NC, Becker K, et al: 2018 Guidelines for the Early Management of $\mathrm{Pa}$ tients with Acute Ischemic Stroke: a Guideline for Healthcare Professionals from the American Heart Association/American Stroke Association. Stroke 2018;49:e46-e110.

6 Troke ST, Roup ST: Tissue plasminogen activator for acute ischemic stroke. The National Institute of Neurological Disorders and Stroke rt-PA Stroke Study Group. N Engl J Med 1995;333:1581-1587.

7 Bamford J, Sandercock P, Dennis M, Warlow C, Burn J: Classification and natural history of clinically identifiable subtypes of cerebral infarction. Lancet 1991;337:1521-1526.

8 Mistry EA, Mistry AM, Nakawah MO, Khattar NK, Fortuny EM, Cruz AS, et al: Systolic blood pressure within 24 hours after thrombectomy for acute Ischemic stroke correlates with outcome. J Am Heart Assoc 2017;6.

9 Robinson TG, Dawson SL, Eames PJ, Panerai RB, Potter JF: Cardiac baroreceptor sensitivity predicts long-term outcome after acute ischemic stroke. Stroke 2003;34:705-711.

10 Sargento-Freitas J, Laranjinha I, Galego O, Rebelo-Ferreira A, Moura B, Correia M, et al: Nocturnalblood pressure dipping in acute ischemic stroke. Acta Neurol Scand 2015;132:323-328.

11 Jain S, Namboodri KKN, Kumari S, Prabhakar S: Loss of circadian rhythm of blood pressure following acute stroke. BMC Neurol 2004;4:1.

12 Okada R, Okada A, Okada T, Nanasato M, Wakai K: Visit-to-visit blood pressure variability is a marker of cardiac diastolic function and carotid atherosclerosis. BMC Cardiovasc Disord 2014;14:188. 